\title{
Temperature-dependent plasticity and fracture mechanism under shear loading in metallic glass
}

\author{
Cen Chen ${ }^{\mathrm{a}, *}$, Bao An Sun ${ }^{\mathrm{b}, \mathrm{c}, *}$, Wei Hua Wang ${ }^{\mathrm{b}, \mathrm{c}}$, Tzu Chiang Wang ${ }^{\mathrm{a}, \mathrm{d}}$ \\ ${ }^{a}$ State Key Laboratory of Nonlinear Mechanics, Institute of Mechanics, Chinese Academy of Sciences, Beijing 100190, PR China \\ ${ }^{\mathrm{b}}$ Institute of Physics, Chinese Academy of Sciences, Beijing 100190, PR China \\ ' Songshan Lake Materials Laboratory, Dongguan, Guangdong 523808, PR China \\ ${ }^{\mathrm{d}}$ School of Engineering Sciences, University of Chinese Academy of Sciences, Beijing 100 049, PR China
}

\section{A R T I C L E I N F O}

\section{Keywords:}

Metallic glass

Shear fracture surface morphology

Temperature

Weibull statistics

Shear transformation zone

Local plastic flow

\begin{abstract}
A B S T R A C T
The fracture surface morphology under shear loading is important for understanding the plasticity and the deformation mechanism of metallic glasses (MGs). However, it is difficult to carry out the shear fracture test for most MGs by conventional test methods due to their limited glass forming ability. In this work, through a unique antisymmetrical four-point bend method, the shear fracture test is carried out under a quasi-static loading at a wide temperature range from $220 \mathrm{~K}\left(0.31 T_{g}, T_{g}\right.$ : glass transition temperature) to $620 \mathrm{~K}\left(0.88 T_{g}\right)$ for a Zr-based MG. Basing on the examination on the fracture surface morphologies, we found three different fracture mechanisms with temperature: at lower temperatures $\left(220 \sim 260 \mathrm{~K}, 0.31 \sim 0.37 T_{g}\right)$, both flower-like and shear-driven vein patterns appear on the fracture surface, which displays a mix of shear- and dilatation- dominated fracture; at intermediate temperatures $\left(300 \sim 570 \mathrm{~K}, 0.42 \sim 0.80 T_{g}\right)$, the shear-driven vein patterns dominate on the fracture surface, indicating the shear-dominated fracture; at higher temperatures $\left(600 \sim 620 \mathrm{~K},>0.80 T_{g}\right)$, a mass of melt droplets indicate the transition from inhomogeneous to homogeneous deformation. From the Weibull statistical analysis on the main fracture features at intermediate temperatures, we found that the size of shear-driven vein patterns increases and the data distribution is sparser with the temperature increasing. The temperature dependence of fracture surface morphology as well as the relationship between the fracture surface feature and the microstructural evolution in MG were also interpreted from the shear transformation zone theory.
\end{abstract}

\section{Introduction}

The plasticity and fracture mechanism of metallic glasses (MGs) have attracted many research attention recently [1-3]. Comparing with the crystalline materials with structural defect, MGs display more complicated fracture and plastic behavior due to their long-range disordered structure [4-6]. Generally, the plastic deformation of MGs is usually inhomogeneous with plastic strain highly localized into narrow (10 20 nm wide) shear bands (SBs) [7,8]. And the local plastic behavior is dominated by the shear-induced disordering at atomic-scale, which is closely related to shear band instability and the fracture behavior of MGs [9]. While, when the temperature is low enough, a transition from shear- to dilatation-dominated failure would replace the failure through SB $[10,11]$. And when the temperature is near to the glass transition temperature, the deformation is approximately homogeneous. Hence, the temperature has a great influence on the fracture and plasticity of MGs [12].
The temperature effect on the fracture and plastic flow mechanism for MGs can be directly revealed by the fracture surface morphologies. For example, the tensile fracture surfaces of Mg-based MG show several types of features at different temperatures, which indicates ductile rupture at lower temperatures $(353 \mathrm{~K}, 373 \mathrm{~K})$ and brittle fracture at higher temperatures $(413 \mathrm{~K})$ [13]. However, the compressive fracture experiments of Zr-based MG display that the vein patterns become sparse on the fracture surface with increasing temperature, which reveals the temperature dependence on local plastic behavior [14]. Hence, it is effective to analyze the plasticity and fracture mechanism by the fracture surface feature for MGs.

It is important that the various fracture surface morphologies of MGs under different loading modes also display a normal/pressure effect on the local plastic flow $[6,15,16]$, which makes it more difficult to understand the fracture mechanism of MGs. Considering that the deformation of MGs under different loading modes could be accomplished by the local arrangement of some atomic clusters which can accommo-

\footnotetext{
* Corresponding authors.

E-mail addresses: chencen@lnm.imech.ac.cn (C. Chen), sunba@iphy.ac.cn (B.A. Sun).
} 
date the shear strain $[3,17]$, the deformation behavior of shear loading, which rules out the normal stress, could reveal the local plastic flow and fracture mechanism for MGs more directly than that of tension and compression.

The shear testing in MGs runs into challenges in terms of traditional mechanical methods due to their forming ability limitation [18]. For MGs, the cylinder sample is the only choice for torsion test [19] and the shear stress distributes linearly along the axial direction of the sample. Since it fails to get a uniform shear stress filed, the experimental analysis of shear fracture surface morphology is difficult to realize. In this paper, we get the shear fracture surface morphologies of a Zr-based MG over a wide temperature range by a unique anti-symmetrical fourpoint bend (anti-FPB) shear test method. The temperature influences on the plasticity and fracture mechanism are revealed based on the experimental results and theoretical analysis. Our results provide insights into the intrinsic deformation and fracture mechanisms of MGs.

\section{Experiments}

The anti-FPB shear test method is suitable to the experimental investigation of shear fracture for macroscopic brittle material [20,21] and has been adopted to investigate the shear fracture behavior for $\mathrm{Zr}$ - and La-based MGs at room temperature [22]. The principle of anti-FPB shear test is shown in Fig. 1, four loading rods are placed anti-symmetrically for a plate sample and the force is transmitted to the loading rods from the press head of the machine (see Fig. 1a). Then, the shear stress field is realized at the anti-symmetrical center since the bending moment is zero in this place, which is confirmed by the illustrations of the bending moment and shear force in Fig. 1b. To get a uniform shear stress field for observing the fracture surface morphologies, the samples are machined with two aligned $90^{\circ} \mathrm{V}$-notches at the anti-symmetrical center [22]. The introduction of two aligned $90^{\circ} \mathrm{V}$-notches at the zero-moment section renders the shear stress distribution quite uniform in that region [23]. We have analyzed the shear stress distribution in the anti-FPB shear samples with different $\mathrm{V}$-notches angles by the finite element method in our early work and found that the shear stress distributes uniformly for the $90^{\circ} \mathrm{V}$-notch samples [22].

The Zr-based MG of $\mathrm{Zr}_{52.5} \mathrm{Cu}_{17.9} \mathrm{Ni}_{14.6} \mathrm{Al}_{10} \mathrm{Ti}_{5}$ (Vit 105) plate samples were prepared by induction melting mixtures of pure metal elements and then casting into $\mathrm{Cu}$ mold. The geometric size of samples is $l=50 \mathrm{~mm}, h=6 \mathrm{~mm}, \delta=2 \mathrm{~mm}$. The samples were machined with two aligned $90^{\circ} \mathrm{V}$-notches at the anti-symmetrical center and the notch depth is $2.0 \mathrm{~mm}$. For each sample, the amorphous structure was confirmed by $\mathrm{X}$-ray diffraction and differential scanning calorimetry. And all samples surfaces were carefully polished with 0.15 um diamond sandpaper.

The samples were tested from $220 \mathrm{~K}\left(0.31 T_{g}\right)$ to $620 \mathrm{~K}\left(0.88 T_{g}\right)$ with 20 40 K temperature interval using the Instron 3384 machine (Norwood, MA). All shear tests were repeated for three times in the same experimental condition with the displacement rate of $0.2 \mathrm{~mm} / \mathrm{min}$ and the heat rate of $10 \mathrm{~K} / \mathrm{min}$. When the test temperature was reached, each sample was held for 15 to 20 min before testing and the environmental cabinet surrounding the test facility was effective to maintain the test temperature in the loading process. After the fracture test, the fracture surfaces of all samples were examined carefully using scanning electron microscopy to get the surface morphologies.

\section{Results}

\subsection{Fracture morphologies of Zr-based $M G$ at different temperatures and transition of fracture mechanism under shear loading}

Fig. 2 shows the shear fracture morphologies of MG at different temperatures. One can see that the main feature of fracture surface changes with temperature, which indicates the transition of deformation mode as temperature is decreasing or increasing. At lower temperatures $\left(0.31 \sim 0.37 T_{g}\right.$, see Fig. $2 \mathrm{a}$ and $\left.\mathrm{b}\right)$, the flower-like patterns
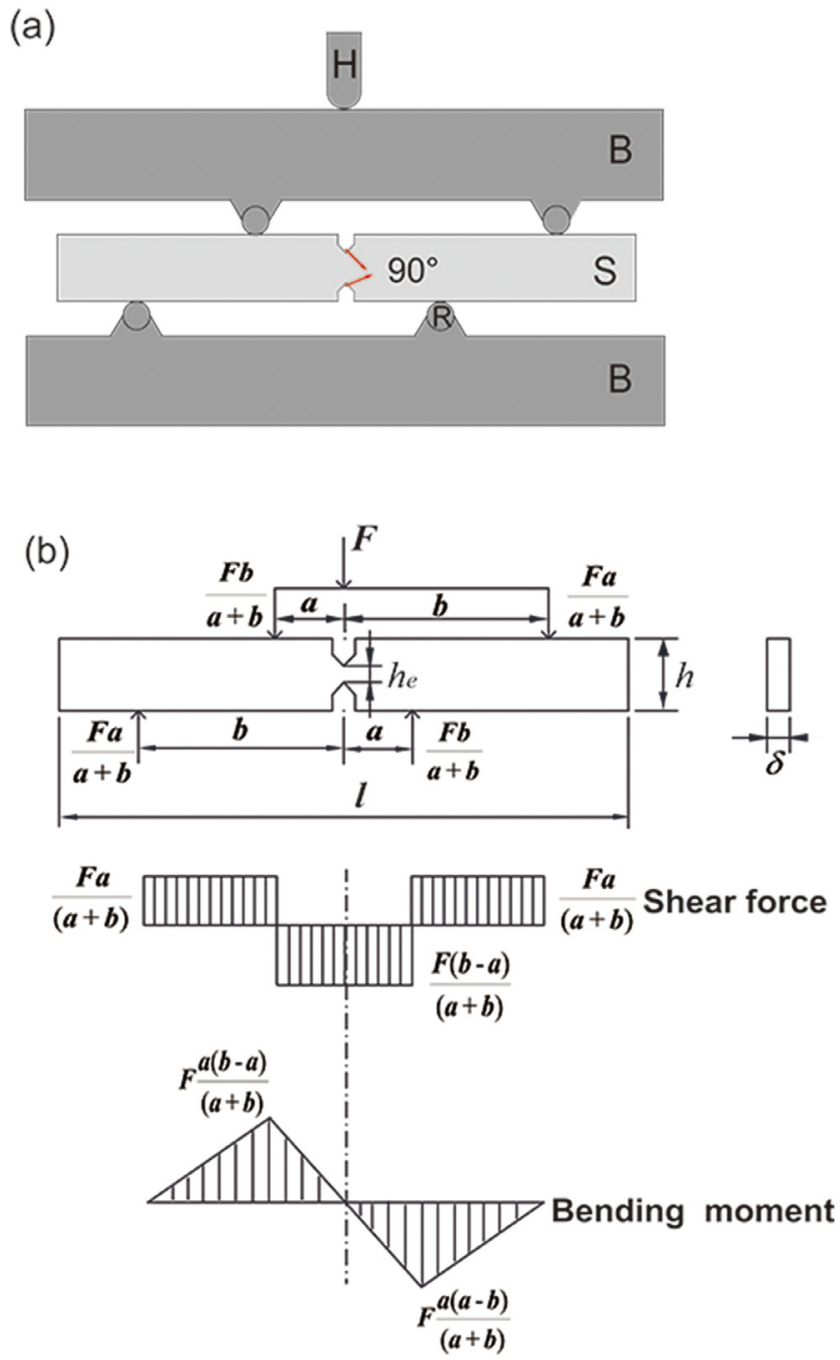

Fig. 1. Anti-FPB shear test. (a) Schematic view of the experiment principle: $H$ is the press head of the machine; $B$ is the grooved block holding the loading rods; $S$ is the sample; $R$ is the loading rod. (b) Diagrams of loading configuration, shear force and bending moment for the sample. $F$ is the force of the machine head; $a$ and $b$ are distances from the anti-symmetrical center to the loading points respectively; $h_{e}$ is the effective height of the reduced cross-section; $\delta$ is the thickness of the sample; $h$ is the effective height of the sample.

appear on the fracture surface accompanied with the shear-driven vein patterns. The existing researches reveal that the flower-like patterns are caused by the dilatation-dominated failure and the dilatation effect has an increasing influence on the failure mode with temperature decrease. Hence, the fracture morphologies from $0.31 T_{g}$ to $0.37 T_{g}$ reveal a mix mode of shear- and dilatation-dominated fracture [10]. At intermediate temperatures $\left(0.42 \sim 0.80 T_{g}\right.$, see Fig. $\left.2 \mathrm{c}-\mathrm{j}\right)$, the shear-driven vein patterns extend along the shear direction and spread over almost the whole fracture surface, which can be attributed to local melting within the main shear band induced by the stored high elastic energy in instantaneous fracture [22]. The molten amorphous exhibiting reduced viscosity within the main SB is easy to flow and results in the formation of shear-driven vein patterns in the fracture surface [3]. And the vein indicates the shear-dominated fracture in SB. At higher temperatures ( $>0.80 T_{g}$, see Fig. $2 \mathrm{k}, 1$ ), a mass of melt droplets are accompanied with the shear-driven vein pattern, which indicates that the temperature rise induced by the adiabatic shear heating may exceed the melting temperature of the MGs and a transition from the inhomogeneous deformation to the homogeneous deformation occurs [12]. 

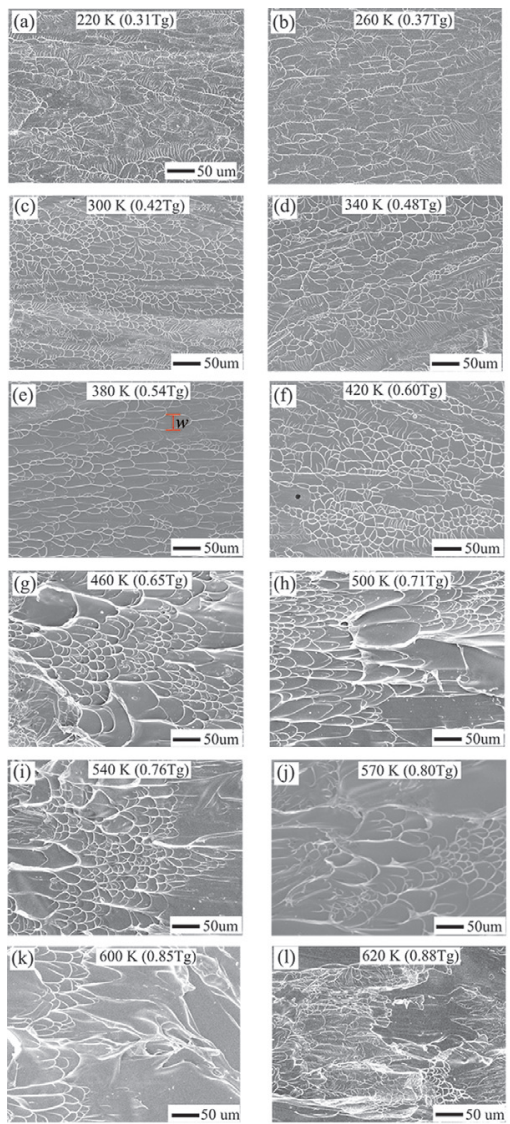

Fig. 2. Shear fracture surface morphologies for Zr-based MG at different temperatures.

\subsection{Weibull statistics for the vein pattern sizes at intermediate temperatures $(0.42 \sim 0.80 \mathrm{Tg})$}

To more deeply understand the temperature effect on the local plastic behavior, the wavelength $w$ (as shown in Fig. 2e) of 300 to 400 veins on different fracture surfaces were measured from $0.42 T_{g}$ to $0.80 T_{g}$, and all measurement data were analyzed by the Weibull statistics. The Weibull statistics is the most popular mathematical description for the fatigue life, time to failure or strength of the material, which had been successfully applied to the failure data and the defects distribution for the MGs $[24,25]$. And the Weibull moduli of samples do not change for the same size and composition. Hence, we adopt it to find the regularities for the distribution of the vein pattern size and their correlation with the plasticity and fracture mechanism.

The distribution function of Weibull statistics [26] is

$F(w)=1-\exp \left[-\left(\frac{w}{\beta}\right)^{\alpha}\right]$

where $\alpha$ is the shape parameter, and $\beta$ is the scale parameter. The shape parameter $\alpha$ governs the shape of the distribution function and thus represents both the "skewness" and the "spread" of the data. For $\alpha$ with larger value, the spread of the data is smaller, which means high uniformity or low variability. Therefore, the shape parameter $\alpha$ can characterize the trend of vein patterns that correlates with the fracture mechanism. The scale parameter $\beta$ determines the scale of the distribution function. For $\beta$ with higher value, the data are more dispersive than that with lower value. Based on the experimental results and Eq.(1), the values for $\alpha$ and $\beta$ at different temperatures are obtained and listed in Table 1 . It is found that the shape parameter $\alpha$ with average value of 3.0 is weakly dependence on the temperature, which indicates that the distribution regularities of the vein pattern sizes are not influenced by temperature. While
Table 1

Shape parameter $\alpha$ and scale parameter $\beta$ of Weibull statistics at intermediate temperatures.

\begin{tabular}{lll}
\hline Temperature $(\mathrm{K})$ & $\alpha$ & $\beta$ \\
\hline 300 & 2.912 & 10.559 \\
340 & 2.697 & 12.608 \\
380 & 2.653 & 13.555 \\
420 & 2.733 & 15.009 \\
460 & 3.555 & 16.831 \\
500 & 2.839 & 17.501 \\
540 & 3.136 & 19.325 \\
570 & 3.312 & 23.057 \\
\hline
\end{tabular}

the scale parameter $\beta$ increases with increasing temperature, which reveals that the distribution of vein pattern sizes is more dispersive at higher temperatures.

The density function of Weibull statistics, which shows the percentage for the number of the vein pattern sizes around a given value $w$ [26], is calculated by

$f(w)=\frac{\alpha}{\beta}\left(\frac{w}{\beta}\right)^{\alpha-1} \exp \left[-\left(\frac{w}{\beta}\right)^{\alpha}\right]$

The density curves for the distribution of the vein pattern sizes at different temperatures could be obtained by Eq. (2), which are displayed with the average values in Fig. 3. It is obvious that the curves follow the same regularity for all temperatures and the peaks of them move to higher values when the temperature rises. Moreover, the data are more centralized at lower temperature and become more dispersive at higher temperatures.

\section{Discussion}

\subsection{Relationship between the transition of fracture mechanism and the activation of shear transformation zone (STZ)}

Since the formation of plastic zone and the fractal nature of dimple structures on the fracture surface for MGs have been explained reasonably based on the STZ theory [5,27], we consider the STZ as the basic deformation unit in the deformation process, which is a small cluster of randomly close-packed atoms that spontaneously and cooperatively reorganize under the applied shear stress [3]. Based on the investigation of the temperature effect on the activation of STZ [3,28-30], the transition of fracture morphologies and mechanism can be explained in a microscopic picture as follows:

At lower temperatures, the activation of an STZ needs to surmount a higher energy barrier [28]. Hence, the STZ is more likely to dilatation operation [10], and the shear operation of STZ will become more difficult with further decrease temperature. Therefore, the fracture mechanism of MGs at lower temperatures is determined by the competition between the shear operation and the dilatation operation of STZ. If the temperature is low enough, the dilatation operation of STZ dominates in the competition. While, if the temperature is not low enough, the shear operation of STZ dominates in the competition. Hence, both of the flower-like and vein patterns appear on the fracture surface from $200 \sim 260 \mathrm{~K}\left(0.31 \sim 0.37 T_{g}\right)$. The flower-like patterns display the dilatation operation of STZ and the vein patterns represent the shear operation of STZ.

At intermediate temperatures $\left(0.42 \sim 0.80 T_{g}\right)$, the activation of STZ is shear-dominated and accompanied by a slight dilatation. The dynamic evolution of the crack can be described as follows: firstly, the shear induced distortion of STZ triggers an avalanche-like behavior of neighboring STZ, which gives rise to a local softening zone and then forms the SBs [31]; then, the fracture occurs in the main SB resulting in a macroscopic shear-dominated failure and the vein patterns form on the fracture surface $[31,32]$. The previous studies consider that more active STZs are 

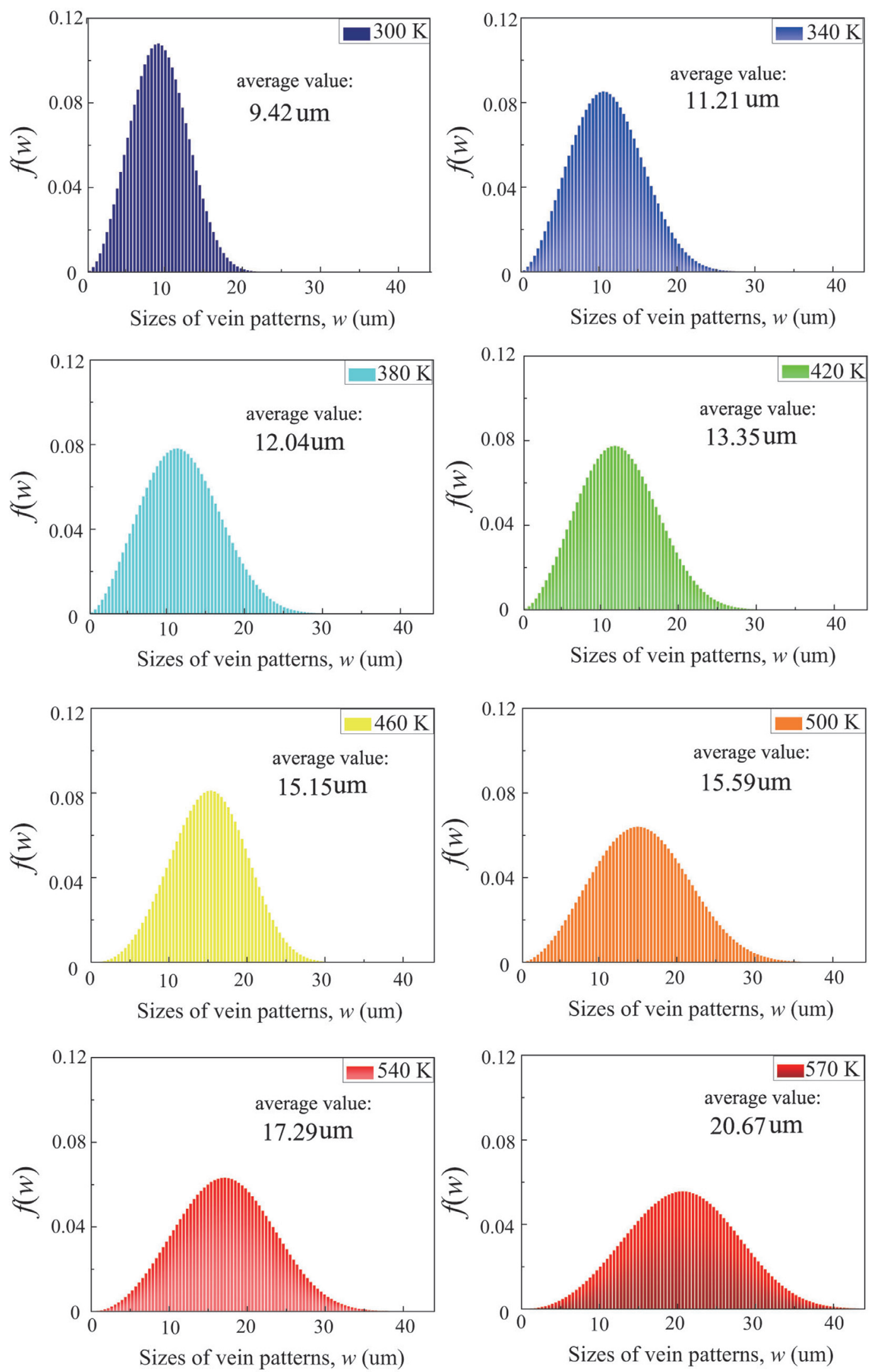

Fig. 3. Density curves for the distributions of the vein pattern sizes on the fracture surface at intermediate temperatures. 


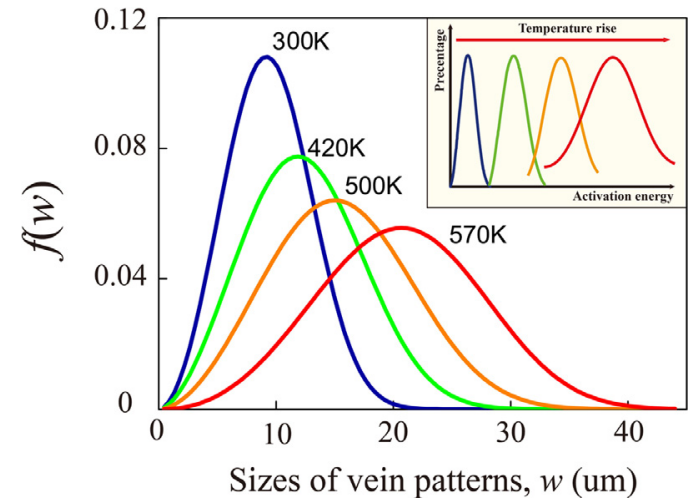

Fig. 4. Comparison for the distribution between the vein pattern sizes and the activation energy spectra of STZs $[33,34]$ from lower to higher temperatures.

organized in one place, larger plastic (softening) zone is formed in this place, the larger dimples are produces on the fracture surface [5,27]. Therefore, the sizes of vein patterns increase with temperature.

With the temperature increase, the STZs become more easy to active, and when the temperature is close to $T_{g}$, a transition from the inhomogeneous deformation (shear banding) to the homogeneous deformation occurs [23]. Hence, a mass of melt droplets appear on the fracture surface at $600 \sim 620 \mathrm{~K}\left(>0.80 T_{g}\right)$.

4.2. Relationship between the vein pattern sizes and the activation energy of STZs at intermediate temperature $(0.42 \sim 0.80 \mathrm{Tg})$

The activation energy spectrum can further confirm the temperature effect on the activation of STZs [33]. The distribution and evolution for the activation energy spectra of MG at different temperatures have been studied by the stress relaxation method and the Kohlrausch-WilliamsWatts (KWW) function [34]. Basing on the previous researches [33,34], we find that the activation energy spectra shift toward the higher value with increasing temperature (insert graph of Fig. 4), which indicates that more STZs with higher energy barriers are activated during the inelastic deformation and the statistical energy barrier distribution of STZs is more dispersive at elevated temperature [34].

From the Weibull statistics, we show the density curves for the distribution of the vein pattern sizes from lower to higher temperatures in Fig. 4, which reveals the plastic zone sizes at the crack tip. Similar to the activation energy spectra of STZs, the value of plastic zone sizes also moves to the higher value with increasing temperature and the distribution is more dispersive at higher temperatures.

Basing on the above analysis, we propose a physical picture for the relationship between the plastic zone size and the activation of STZs in Fig. 5. With temperatures increase, the total energy required for the activation of STZs and the characteristic volume of STZs decrease because the STZs occur everywhere in the sample and their movement becomes easy [30], then it naturally follows that the STZs should be easier to activate with the increasing temperature [28]. The plastic zone sizes become larger since the STZs are easier to activate and the number of active STZs increases with increasing temperature, and then the vein pattern sizes are larger at higher temperature. And since the energy barrier distribution of STZs is more dispersive at elevated temperature, the distribution of the vein pattern sizes is sparser with the increasing temperature.

\section{Conclusion}

The plasticity and fracture mechanism under shear loading at different temperatures in a Zr-based MG has been investigated by a unique anti-FPB method. With increasing testing temperature from $220 \mathrm{~K}\left(0.31 T_{\mathrm{g}}\right)$ to $620 \mathrm{~K}\left(0.88 T_{\mathrm{g}}\right)$, the different features on the fracture surfaces indicate a transition of the microscopic plasticity and fracture mechanism for MG, which can be analyzed by the activation of STZs as: at lower temperatures $\left(0.31 \sim 0.37 T_{g}\right)$, the STZs are more likely to dilatation operation, the fracture mechanism depends on the competition result between the shear operation and dilatation operation of STZs; at intermediate temperatures $\left(0.42 \sim 0.80 T_{\mathrm{g}}\right)$, the STZs are shear-dominated, and the increase of the vein pattern sizes with temperature reflects the temperature dependence of local plastic behavior; at higher temperatures $\left(>0.80 T_{\mathrm{g}}\right)$, a transition from the inhomogeneous deformation (shear banding) to the homogeneous deformation occurs. Our study reveals the temperature effect on the intrinsic deformation behavior of MG and offers a new foundation for the engineering applications for MG.

\section{Declaration of Competing Interest}

The authors declare that they have no known competing financial interests or personal relationships that could have appeared to influence the work reported in this paper.

\section{Acknowledgements}

This work was supported by the National Natural Science Foundation of China [Grant Nos. 11702295, 11790292, 11532013, 11790291]

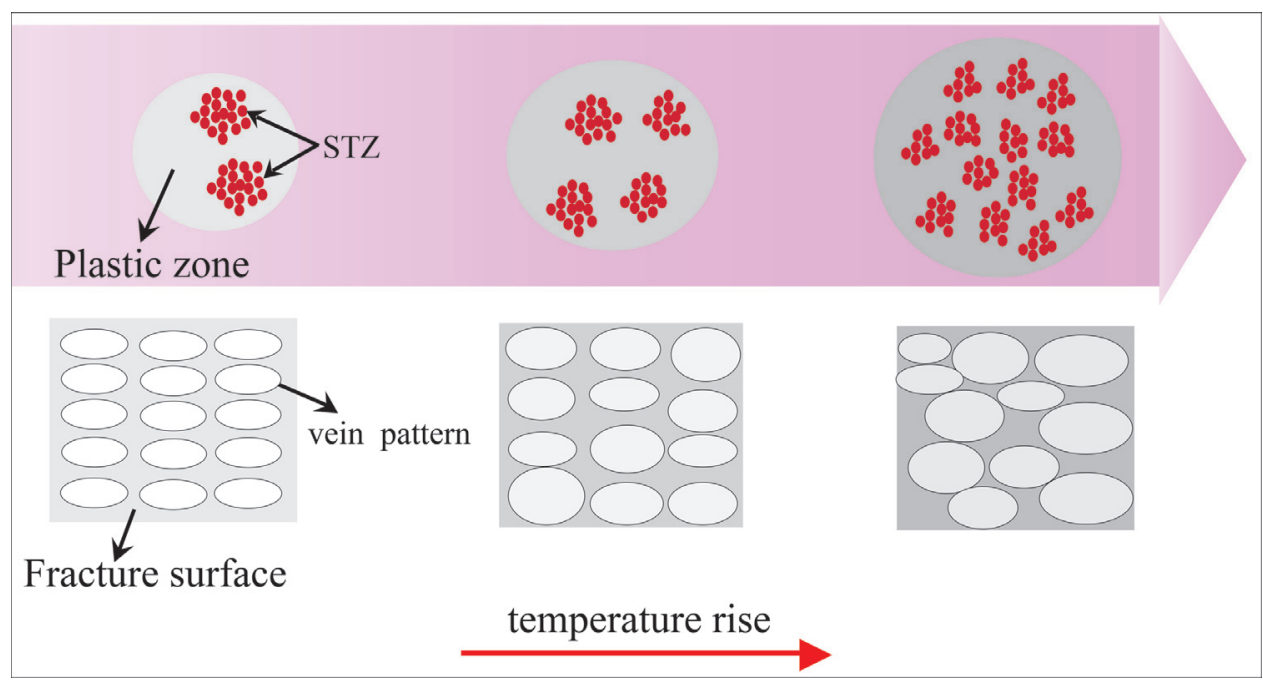

Fig. 5. Relationship between the plastic zone size (vein pattern size) and the activation of STZs with temperature rise. 
and the Strategic Priority Research Program of the Chinese Academy of Sciences[Grant No. XDB22040503 and XDPB0601], the National Key Research and Development Plan [Grant No. 2016YFB0300501 \& 2017YFB0903902], and the Key Research Program of Frontier Sciences [QYZDY-SSW-JSC017]. B. A. Sun thanks the support from National Natural Science Foundation of China [Grant Nos. 51822107, 51671121, 51761135125] and National Key Research and Development Plan [Grant No. 2018YFA0703603] and the Natural Science Foundation of Guangdong Province [Grant No. 2019B030302010].

\section{References}

[1] J.J. Lewandowski, W.H. Wang, A.L. Greer, Intrinsic plasticity or brittleness of metallic glasses, Phil. Mag. Lett. 85 (2005) 77-87.

[2] M. Zink, K. Samwer, W.L. Johnson, S.G. Mayr, Plastic deformation of metallic glasses: size of shear transformation zones from molecular dynamics simulations, Phys. Rev. B. 73 (2006) 172203.

[3] A.S. Argon, Plastic deformation in metallic glasses, Acta. Metall. 27 (1979) 47-58.

[4] Z.F. Zhang, G. He, J. Eckert, L. Schultz, Fracture mechanisms in bulk metallic glassy materials, Phys. Rev. Lett 91 (2003) 318-322.

[5] M. Gao, B.A. Sun, C.C. Yuan, J. Ma, W.H. Wang, Hidden order in the fracture surface morphology of metallic glasses, Acta. Mater 60 (2012) 6952-6960.

[6] R.T. Qu, Z.F. Zhang, Compressive fracture morphology and mechanism of metallic glass, J. Appl. Phys. 114 (2013) 193504.

[7] J. Fornell, A. Concustell, S. Suriñach, W.H. Li, N. Cuadrado, A. Gebert, M.D. Baró, J. Sort, Yielding and intrinsic plasticity of Ti-Zr-Ni-Cu-Be bulk metallic glass, Int. J. Plast. 25 (2009) 1540-1559.

[8] Y. Chen, M.Q. Jiang, L.H. Dai, Collective evolution dynamics of multiple shear bands in bulk metallic glasses, Int. J. Plast. 50 (2013) 18-36.

[9] B.A. Sun, W.H. Wang, The fracture of bulk metallic glasses, Prog. Mater. Sci. 74 (2015) 211-307.

[10] M.Q. Jiang, G. Wilde, J.H. Chen, C.B. Qu, S.Y. Fu, F. Jiang, L.H. Dai, Cryogenic-temperature-induced transition from shear to dilatational failure in metallic glasses, Acta. Mater 77 (2014) 248-257.

[11] X.L. Bian, G. Wang, J. Yi, Y.D. Jia, J. Bednarčík, Q.J. Zhai, I. Kaban, B. Sarac, M. Mühlbacher, F. Spieckermann, J. Keckes, J. Eckert, Atomic origin for rejuvenation of a Zr-based metallic glass at cryogenic temperature, J. Alloys. Comp. 718 (2017) 254-259.

[12] F. Spaepen, A microscopic mechanism for steady state inhomogeneous flow in metallic glasses, Acta. Metall. 25 (1977) 407-415.

[13] L.A. Deibler, J.J. Lewandowski, Effects of changes in strain rate and test temperature on $\mathrm{Mg}_{85} \mathrm{Ca}_{5} \mathrm{Cu}_{10}$ metallic glass ribbons, Mater. Sci. Eng. A. 527 (2010) 2214-2221.

[14] Y. Huang, W. Zheng, F. He, J. Shen, The temperature dependent dynamic mechanical response of a ZrCuNiAl bulk metallic glass, Mater. Sci. Eng. A. 551 (2012) 100-103.
[15] R.T. Qu, M. Stoica, J. Eckert, Z.F. Zhang, Tensile fracture morphologies of bulk metallic glass, J. Appl. Phys. 108 (2010) 063509.

[16] G. Wang, D.Q Zhao, H.Y. Bai, M.X. Pan, A.L. Xia, B.S. Han, X.K. Xi, Y. Wu, W.H. Wang, Nanoscale periodic morphologies on the fracture surface of brittle metallic glasses, Phys. Rev. Lett. 98 (2007) 235501.

[17] M.H. Cohen, D. Turnbull, Molecular transport in liquids and glasses, J. Chem. Phys. 31 (1959) 1164-1169.

[18] D.D. Liang, X.S. Wei, C.T. Chang, J.W. Li, X.M. Wang, J. Shen, Effect of W addition on the glass forming ability and mechanical properties of Fe-based metallic glass, J. Alloys. Comp. 731 (2018) 1146-1150.

[19] B. Sarac, F. Spieckermann, A. Rezvan, C. Gammer, L. Krämer, J.T. Kim, J. Keckes, R. Pippan, J. Eckert, Annealing-assisted high-pressure torsion in $\mathrm{Zr}_{55} \mathrm{Cu}_{30} \mathrm{Al}_{10} \mathrm{Ni}_{5}$ metallic glass, J. Alloys. Comp. 784 (2019) 1323-1333.

[20] D.E. Walrath, D.F. Adams, The losipescu shear test as applied to composite materials, Exp. Mech. 23 (1983) 105-110.

[21] J. Morton, H. Ho, M.Y. Tsai, G.L. Farley, An evaluation of the Iosipescu specimen for composite materials shear property measurement, J. Com. Mater. 26 (1992) 708-750.

[22] C. Chen, M. Gao, C. Wang, W.H. Wang, T.C. Wang, Fracture behaviors under pure shear loading in bulk metallic glasses, Sci. Rep. 6 (2016) 39522.

[23] N. losipescu, New accurate procedure for single shear testing of metals, J. Mater. 2 (1967) 537-566.

[24] M. Calvo, Application of the Weibull statistics to the characterization of metallic glass ribbons, J. Mater. Sci. 24 (1989) 1801-1808.

[25] F. Farahani, R. Gholamipour, Statistical weibull analysis of compressive fracture strength of $\left(\mathrm{Zr}_{55} \mathrm{Cu}_{30} \mathrm{Al}_{10} \mathrm{Ni}_{5}\right)_{99} \mathrm{Nb}_{1}$ bulk metallic glass, J. Alloys. Comp. 695 (2017) 2740-2744.

[26] Y.Y. Zhao, E. Ma, J. Xu, Reliability of compressive fracture strength of Mg-Zn-Ca bulk metallic glasses: flaw sensitivity and Weibull statistics, Scripta. Mater. 58 (2008) 496-499.

[27] J.S. Langer, Dynamics of shear-transformation zones in amorphous plasticity: formulation in terms of an effective disorder temperature, Phys. Rev. E. 70 (2004) 041502

[28] K.E. Prasad, R. Raghavan, U. Ramamurty, Temperature dependence of pressure sensitivity in a metallic glass, Scripta. Mater. 57 (2007) 121-124.

[29] J.S. Langer, Shear-transformation-zone theory of plastic deformation near the glass transition, Phys. Rev. E 77 (2008) 021502.

[30] K.E. Prasad, U. Ramamurty, Effect of temperature on the plastic zone size and the shear band density in a bulk metallic glass, Mater. Sci. Eng. A. 535 (2012) 48-52.

[31] A.S. Argon, Strain avalanches in plasticity, Phil. Mag. 93 (2013) 3795-3808.

[32] X.K. Xi, D.Q. Zhao, M.X. Pan, W.H. Wang, Y. Wu, J.J. Lewandowski, Fracture of brittle metallic glasses: brittleness or plasticity, Phys. Rev. Lett. 94 (2005) 125510.

[33] W. Jiao, B.A. Sun, P. Wen, H.Y. Bai, Q.P. Kong, W.H. Wang, Crossover from stochastic activation to cooperative motions of shear transformation zones in metallic glasses, Appl. Phys. Lett. 103 (2013) 3113-R.

[34] W. Jiao, P. Wen, H.L. Peng, H.Y. Bai, B.A. Sun, W.H. Wang, Evolution of structural and dynamic heterogeneities and activation energy distribution of deformation units in metallic glass, Appl. Phys. Lett. 102 (2013) 080901. 